Sinha Pallavi

Sinha Bimal Kumar

Baskota Dharma Kanta

Neupane Yogesh

Acharya Kunjan

Ganesh Man Singh Memorial

Academy of ENT - Head \& Neck

Studies, Tribhuvan University Teaching

Hospital, Institute of Medicine,

Kathmandu, Nepal.

Correspondence to:

Dr Pallavi Sinha

Department of ENT - HNS

Ganesh Man Singh Bhawan

TU Teaching Hospital,

Kathmandu, Nepal.

email: dr_pallavi@hotmail.com

\section{TREATMENT OUTCOMES OF LARYNGEAL AND HYPOPHARYNGEAL SQUAMOUS CELL CARCINOMAS IN A TERTIARY CARE CENTER OF NEPAL}

Aims And Objectives:

To analyze the treatment outcomes in patients with laryngeal and hypopharyngeal cancers using locally recurrent or residual disease as an outcome measure.

Materials And Methods:

Data for the observational study was obtained from the Cancer Registration Forms and Register of the Department of E.N.T. and Head Neck Surgery of T.U. Teaching Hospital, Maharajgunj. Patients with histopathologically proven squamous cell carcinomas of larynx and hypopharyx with a minimum follow up duration of 6 months were included in the study. Treatment outcomes were assessed as patients having recurrent/ residual disease or not. Data was analysed using percentages.

Results:

Of the 74 patients included in the study $12 \%$ were females and $88 \%$ were males. The mean age was 60.82 years. The mean follow up duration was 37.7 months. Majority, $38 \%$ had carcinoma glottis. $59.5 \%$ of the patients were detected in the advanced stage of disease. Overall, recurrenct/ residual tumour was seen in $13.3 \%$ of the early stage disease group whereas recurrent or residual disease was seen in $25 \%$ of the advanced stage tumour group. Recurrent/ residual disease was found in $31.8 \%$ of the patients that were treated with CT/RT as their primary treatment modality.

Conclusion:

Recurrence rate for squamous cell carcinomas of the larynx and hypopharynx in early as well as advanced stages was found to be higher in the current study as compared to the data available from other parts of the world. The rate was observed to be higher in those treated with chemoradiation as their primary treatment modality. This leads to the question as to whether treatment modalities other than chemoradiotherapy should be adopted especially in the treatment of early laryngeal and hypopharyngeal cancers. Conservative endolaryngeal surgery may be a good alternative to radiotherapy for the treatment of early laryngeal cancers. However, a larger multicentric study needs to be conducted.

Ke y w or d s: Squamous cell carcinoma, Chemoradiation, Recurrence.

\section{INTRODUCTION:}

Head and Neck malignancies are fairly common globally. They are the sixth leading cause of cancer-related mortality worldwide1. Incidence of head and neck cancer varies with geography with high rates being reported in France, India, South America and Eastern Europe2,3. In the Indian subcontinent more than $45 \%$ of all cancers arise in the head and neck 4 . Among the malignancies arising in the head and neck region more than $90 \%$ are squamous cell type. In most regions of the world majority of cancers arise in the laryn $x^{1,2}$. The treatment of laryngeal cancers requires a multidisciplinary approach and depends on various factors like patient factors, tumour factors and the healthcare facilities available. For example the general health condition of the patient, the patient's pulmonary reserve, the site and stage of the tumour, the expertise of the treating surgeon, the radiotherapy and rehabilitation facilities available, all play a vital role in the decisionmaking process for the treatment of laryngeal and hypopharyngeal cancers. The modalities of treatment currently available in our set-up are chemoradiotherapy, surgery or combined therapy. Patients with early low volume tumours are referred for radiotherapy with organ preservation as one of the main objectives. Patients with advanced but operable tumours undergo surgery which may or may not be followed by chemoradiotherapy depending upon the post-operative histopathology report. Patients with advanced stage tumours which are not operable are referred for palliative chemoradiotherapy. The aims and objectives of this study were to analyze the treatment outcomes in patients with laryngeal and hypopharyngeal cancers using locally recurrent or residual disease as an outcome measure and also to note in locally recurrent or residual tumours, the primary treatment modality and the stage of tumour prior to treatment. Such a study has not been carried out in Nepal and it can be used as a platform for future research. The results obtained from this study can be compared with the data available from other parts of the world.

\section{MATERIALS AND METHODS:}

The data for this observational study was obtained from the Cancer Registration Forms and Register of the Department of E.N.T. and Head Neck Surgery of T.U. Teaching Hospital, Maharajgunj, Kathmandu, Nepal. Patients attending the Ganesh Man Singh Memorial Academy for ENT and Head Neck Studies, in as well as out patient clinics with histopathologically proven squamous cell carcinomas of larynx were included in this study. A minimum follow up duration of 6 months was required for inclusion. Those patients whose records were not complete or those who had not followed up after being diagnosed were excluded from the study.
Tumour staging was done as per TNM staging of UICC 2009. T1 and $\mathrm{T}_{2}$ stages were taken as early stage disease and $\mathrm{T}_{3}$ and $\mathrm{T}_{4}$ were taken as advanced stage disease.

Treatment outcomes were assessed as patients having recurrent/ residual disease or not having recurrent/ residual disease after at least 6 months of completion of the primary treatment modality. If after 6 months of completion, a suspicious lesion was noticed then biopsy was taken. If the lesion was histopathologically positive for squamous cell carcinoma then it was labeled as recurrent or residual tumour. Data was analysed using percentages.

\section{RESULTS:}

Records of 131 patients of squamous carcinomas of larynx and hypopharynx were available. However, only 74 fulfilled the inclusion criteria and were included in the study. There were $12 \%(9)$ females and $88 \%(65)$ males. The patients included in the study were in the age range of 41-80 years with a mean age 60.82 years. The minimum follow up duration was 6 months and the maximum 192 months. Mean follow up duration was 37.7 months. Among the patients included, majority (38\%) were diagnosed as carcinoma glottis whereas trans-glottic carcinoma was the least common being found in only $17 \%$ of the cases.

Fig. 1: Distribution according diagnosis

Diagnosis
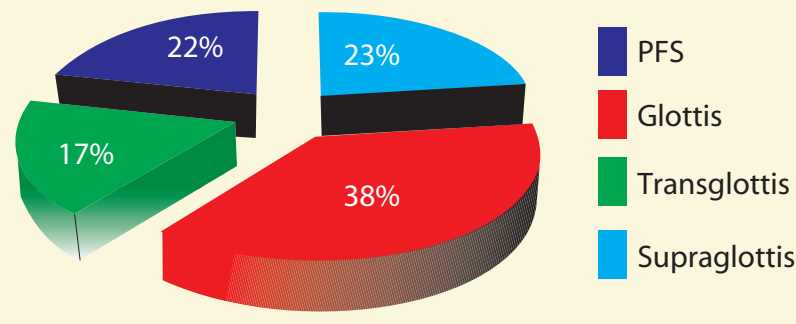

Maximum number of patients (40/74) that is $54 \%$ were diagnosed at T3 stage of tumour whereas minimum (4/74) i.e. $5.4 \%$ number of patients were diagnosed at $\mathrm{T} 4$ stage. $59.5 \%$ of the patients were 
detected in the advanced stage of disease i.e. $\mathrm{T}_{3}$ and $\mathrm{T}_{4}$ Out of the total 11 patients with T1 stage of disease, recurrence was seen in 3 that is $27.3 \%$ of the patients. Out of the total 19 patients with $T_{2}$ stage of disease, recurrence was seen in 2 that is $10.5 \%$ of the patients. Out of the total 40 patients with T3 stage of disease, recurrence was seen in 10 that is $25 \%$ of the patients. Out of the total 4 patients with T4 stage of disease, recurrence was seen in 1 that is $25 \%$ of the patients. Overall, recurrenct/ residual tumour was seen in $13.3 \%$ of the early stage disease group whereas recurrent or residual disease was seen in $25 \%$ of the advanced stage tumour group.

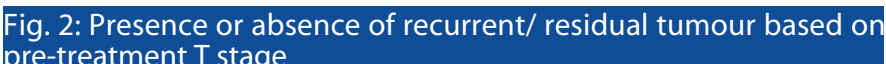
pre-treatment T stage

Outcome based on T stage of tumour

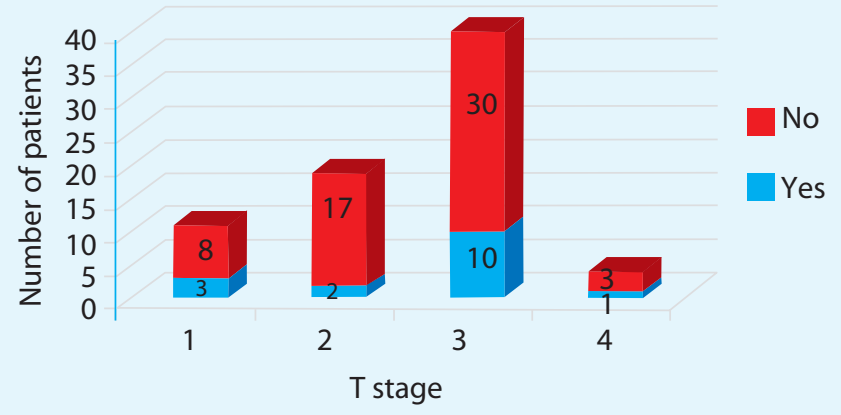

Recurrent/ residual disease was found in 14 patients that is $31.8 \%$ of the patients that were treated with $\mathrm{CT} / \mathrm{RT}$ as their primary treatment modality. There was no recurrent/ residual disease in patients treated with Surgery alone as their treatment modality. There was found to be recurrent/ residual disease in $13.3 \%$ of patients treated with surgery along with adjuvant chemoradiotherapy

Fig. 3: Presence or absence of recurrent/ residual disease based on primary treatment modality

Recurrent/Residual Disease

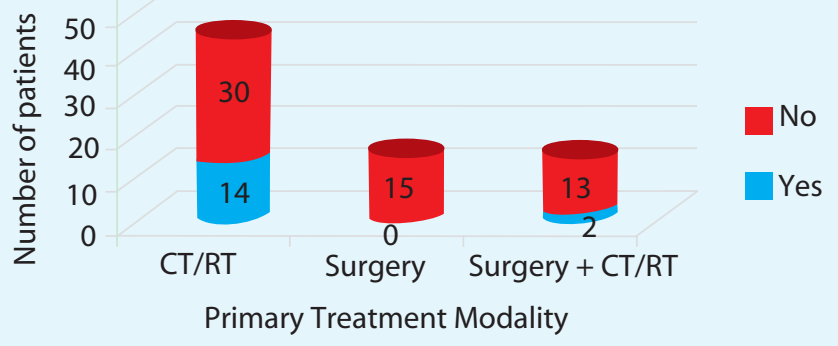

DISCUSSION:

We frequently come across patients with laryngeal and hypopharyngeal cancers in our day to day practice and we manage patients based on the international norms set. However, an analysis of the treatment outcomes in our set-up has never been carried out. With such an analysis we can find out whether there is any room for improvement in our methods of treatment of laryngeal and hypopharyngeal cancers and whether there are any modifications that we need to make. In the current study, patients with a minimum follow up duration of 6 months after completion of their primary treatment modality were included. An interval of 6 months was allowed for post-operative or post radiotherapy changes to settle down as at times it is very difficult to differentiate between post radiotherapy changes and recurrent disease. More number of males were found to be suffering from laryngeal and hypopharyngeal cancers as compared to females. This is similar to results obtained in U.K. in 20095 . The mean age of patients in this study was 60.82 years which is in accordance with other data available which states that laryngeal and hypopharyngeal cancers are rare before the age of 40 years and the incidence is strongly associated with age $6.38 \%$ of the patients were diagnosed as carcinoma glottis. According to U.K. cancer data carcinoma of the glottis is the most common among the subsites of the larynx found in $45 \%$ of the patients 5 . Head and neck cancer patients usually present with late-stage, locally advanced disease. $59.5 \%$ of the patients presented at an advanced stage of disease which is similar to the results in the study by Awada and Castro in which around 75\% of patients were diagnosed with stage III-IV1. Disease recurrence was found in $27.3 \%$ of the patients with T1 stage of tumour which is high as compared to the results of $75-100 \%$ locoregional control obtained by Dickens et al7. 25\% disease recurrence/ residual disease found in advanced stage disease which is high as compared to 2-12\% primary recurrence found in study by Wolf et al8. The rate of recurrent or residual tumours was found to be highest among those who underwent chemoradiotherapy. The results obtained in the current study are inferior to those from other parts of the world especially in the treatment outcome of T1 tumours. Many radiotherapy cohort studies report excellent success rates for early laryngeal cancer ${ }^{9}$. On examining the literature endoscopic laryngeal surgery and radiotherapy seem to have very similar cure rates 10,11 . From the results it can be also be stated that surgery in this set-up provides a better control rate for disease as compared to chemoradiotherapy. There are no randomized controlled trials (RCT) comparing different surgical treatments for advanced laryngeal cancer, but the Veteran's Administration Trial and text books such as Weinstein et al recommend total laryngectomy with voice rehabilitation for $\mathrm{T}_{4}$ tumours and are in clinical equipoise between laryngectomy and radiotherapy for T3 tumours8,12.

\section{CONCLUSION:}

Recurrence rate for squamous cell carcinomas of the larynx and hypopharynx in early as well as advanced stages was found to be higher in the current study as compared to the data available from other parts of the world. The rate was observed to be higher in those treated with chemoradiation as their primary treatment modality. This leads to the question as to whether treatment modalities other than chemoradiotherapy should be adopted especially in the treatment of early laryngeal and hypopharyngeal cancers. Conservative endolaryngeal surgery may be a good alternative to radiotherapy for the treatment of early laryngeal cancers. However a larger multicentric study needs to be conducted.

\section{REFERENCES:}

1. Awada A, Castro GD. Head and neck cancer emerging strategies: advances and new challenges. Current Opinion in Oncology 2009, 21:191-3

2. Johnson NW. Oral cancer: a worldwide problem. FDI World 1997; 6: 19-21.

3. Moore SR, Johnson NW, Pierce AM, Wilson DF. The epidemiology of mouth cancer: a review of global incidence. Oral Diseases 2000; 6: 65-74

4. Sankarnarayanan R. Oral cancer in India: a clinical and epidemiological review. Oral Surgery, Oral Medicine and Oral Pathology 1990; 69: 325-30

5. Cancer Research UK website. 2009 http://info.cancerresearchuk.org/cancerstats/types/larynx/acces sed October 5, 2009.

6. Mackenzie K, Mehanna H. Larynx. Stell and Maran's Textbook of Head and Neck Surgery and Oncology. 5th ed. Hodder Arnold 2012. 645-60.

7. Dickens WJ, Cassisi NJ, Million RR et al. Treatment results of early vocal cord carcinoma: A comparison of apples and oranges. Laryngoscope 1983; 93: 216-219.

8. The Department of Veterans Affair Cancer Study Group. Induction chemotherapy plus radiation compared with surgery plus radiation in patients with advanced laryngeal cancer. N Engl J Med 1991; 324: $1685-90$

9. Inoue $\mathrm{T}$, Inoue $\mathrm{T}$, Ikeda $\mathrm{H}$, Teshima $\mathrm{T}$, Yamazaki $\mathrm{H}$, Murayama $\mathrm{S}$, Othani M, Ozeki S. Comparison of early glottic and supraglottic carcinoma treated with conventional fractionation of radiotherapy. Strahlenther Onkol. 1993 Oct;169(10):584-9

10. Moreau PR. Treatment of laryngeal carcinomas by laser endoscopic microsurgery. Laryngoscope 2000;110: 1000-6

11. Ambrosch $P$, Kron M, Steiner W. Carbon dioxide laser microsurgery for early supraglottic carcinoma. Annals of Otology, Rhinology and Laryngology 1998; 107:680-8

12. Weinstein GS, Brasnu 0, Laccourreye H. Organ preservation surgery of the larynx. California: Singular Press. 